\title{
СБАЛАНСИРОВАННЫЕ БЛОКИ ПОКАЗАТЕЛЕЙ, ОБРАЗУЮЩИЕ СИСТЕМУ ОЦЕНКИ ДЕЯТЕЛЬНОСТИ ТОРГОВОЙ ОРГАНИЗАЦИИ
}

\author{
(C) 2021 Воронцов В.Г. \\ студент \\ Финансовый университет при Правительстве Российской Федерации, Россия, Москва \\ ООО «ТПК «Белтимпэкс, Россия, Москва \\ E-mail:vg.vorontsov@mail.ru
}

(c) 2021 Петров A.M.

доктор экономических наук, профессор Департамента бизнес-аналитики факультета налогов, аудита и бизнес-анализа, главный научный сотрудник Центра анализа, управления рисками и внутреннего контроля в цифровом пространстве

Финансовый университет при Правительстве Российской Федерации, Россия, Москва E-mail: palmi@inbox.ru

Нестабильная экономическая ситуация, динамично изменяющийся рынок, высокая конкуренция в торговой отрасли вынуждают предприятие постоянно адаптироваться к новым условиям. В условиях неопределенности перед торговым предприятием особенно остро стоит вопрос выбора рыночной и управленческой стратегии, от успеха реализации которой зависит положение предприятия на рынке и его конечная финансовая результативность. Решить эту проблему помогает системный подход, базирующийся на сбалансированной системе показателей предприятия.

Ключевые слова: система сбалансированных показателей, финансы, бизнес-процессы, обучение персонала, система оценки, риски

Для оценки деятельности любого коммерческого предприятия его необходимо рассматривать с точки зрения системного подхода, позволяющего подходить комплексно к процессу управления и организации основных экономических процессов. Системный подход базируется на сбалансированной системе показателей предприятия, под которой принято понимать систему стратегического управления коммерческой структурой на основе измерения, анализа и оценки ее эффективности и результативности по определенному набору логически подобранных показателей, отражающих все финансовые и нефинансовые аспекты деятельности анализируемого хозяйствующего субъекта.

Сбалансированная система показателей предприятия (ССП) является важнейшим инструментом в реализации стратегии коммерческой структуры. Суть ССП заключается в рамках четырех перспектив предприятия:

- финансы;

- взаимоотношения с клиентами (рынок);

- внутренние бизнес-процессы;

- обучение (развитие) и повышение квалификации персонала.

Совокупность данных перспектив обеспе- чивает предприятию целенаправленный мониторинг его финансово-хозяйственной, коммерческой и управленческой деятельности в целях предупреждения возникающих проблем и затруднений, а также прогнозирования последствий от принятия тех или иных решений в процессе деятельности предприятия.

Рационально организованная система ССП открывает перед предприятием определенные перспективы и позволяет:

- сконцентрировать имеющиеся в распоряжении предприятия ресурсы (технологические, финансовые, информационные, трудовые и т.д.) на реализации стратегической концепции, приблизив к достижению поставленных целей и задач;

- направить рутинную каждодневную работу производственных, коммерческих, кадровых, административных структур предприятия по направлению достижения стратегических целей хозяйствующего субъекта;

- повысить процесс управляемости всеми процессами, происходящими на предприятии;

- снизить/минимизировать риски в процессе жизнедеятельности торговой компании.

Таким образом, система сбалансирован- 
ных показателей позволяет объединить процесс и уровень оперативного и стратегического управления. Процесс внедрения ССП в практику деятельности торгового предприятия довольно трудоемок и сложен. Однако, несмотря на кажущуюся сложность и многоступенчатость, ССП позволяет предприятию объединить сразу несколько целей в единую стратегию, что непосредственно сказывается на конечной хозяйственной результативности торгового предприятия.

Сбалансированная система показателей Р. Каплана и Д. Нортона, основанная на взаимосвязи взаимодействии четырех перспектив представлена на рисунке 1.

Перспектива финансов торгового предприятия предполагает обзор и расчет финансовых показателей, характеризующую конечную результативность коммерческой структуры. Традиционно важнейшими финансовыми показателями деятельности предприятия является чистая операционная прибыль, рентабельность, генерируемый денежный поток и т.д. [1, с.338].

Перспектива клиентов включает показатели, отражающие конкурентоспособность торгового предприятия, его конкурентные преимущества и рыночные позиции относительно клиентов. Рассматриваемая перспектива в реалиях современной экономической действительности является очень важной в деятельности торгового предприятия. Прежде всего, это объясняется тем, что в условиях насыщенности рынка предприятиям, особенно торгового профиля, все сложнее выдерживать конкуренцию, находить новые рынки сбыта и завоевывать доверие новых покупателей. В связи с этим данная перспектива в системе сбалансированных показателей является очень важной, ведь именно от нее зависит достижение финансовых показателей коммерческой структуры.

К «клиентским» показателям можно отнести общая удовлетворенность клиентов ока-

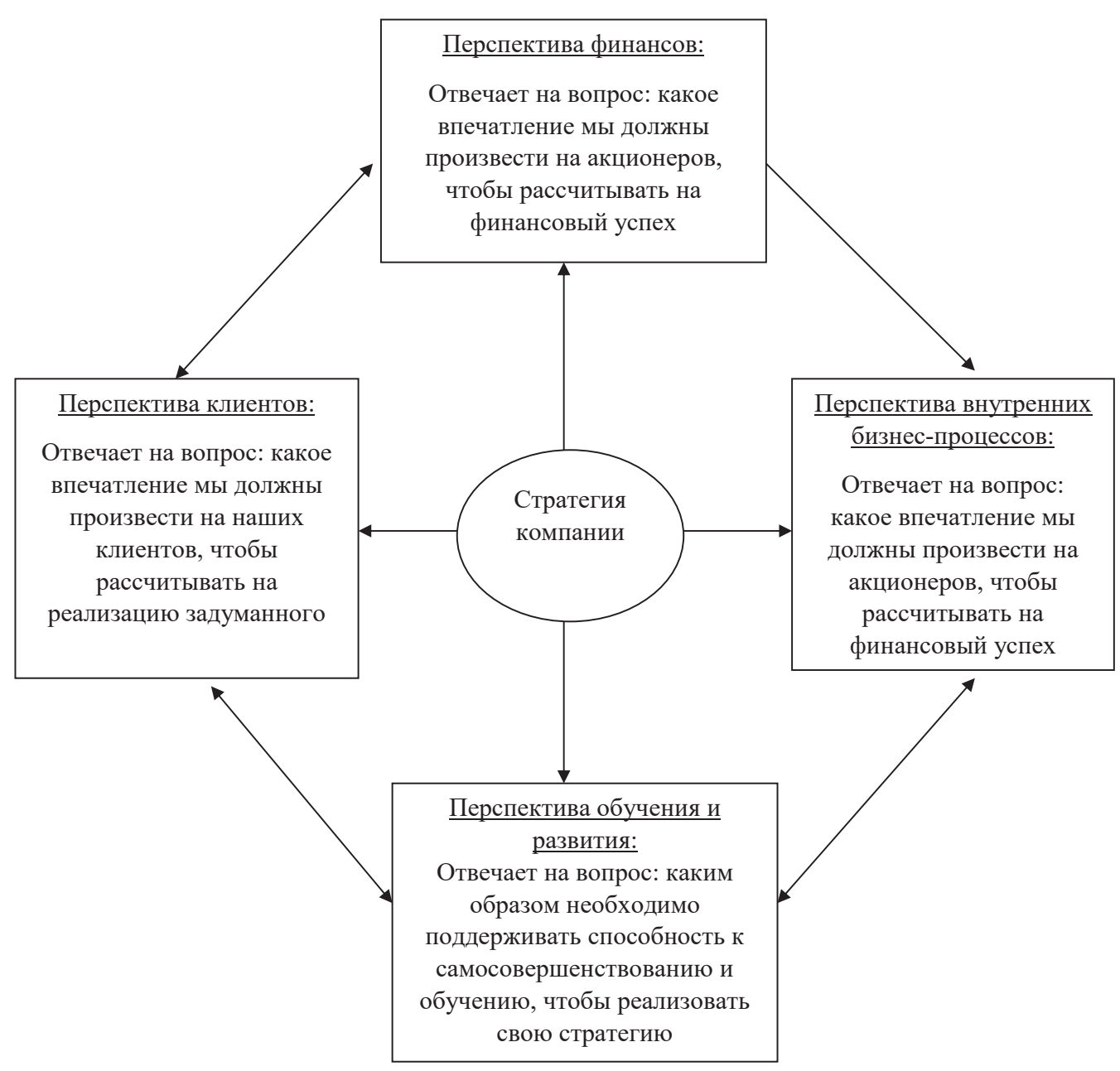

Рисунок 1. Взаимодействие перспектив системы сбалансированных показателей 
зываемыми торговыми услугами, качеством реализуемого товара, доля рынка, занимаемая предприятием, доля новых привлеченных клиентов. Кроме того, такой показатель как удовлетворенность клиентов должен формироваться на основе оценки промежуточных показателей, таких как, например, сроки доставки, доля брака и сроки ответа на рекламации и т.д. Клиентская составляющая позволяет компании с помощью реализации стратегии, ориентированной на клиента, достичь целевых финансовых показателей.

Перспектива внутренние бизнес-процессы предприятия характеризуется показателями бизнес-процессов, необходимых для удовлетворения потенциальных и реальных клиентов торгового предприятия. Показатели, составляющие анализируемую перспективу ССП, связаны с разработкой продукции или реализуемой услуги, внедрением инновационных процессов и научно-технических достижений на любых этапах деятельности хозяйствующего субъекта.

Показатели ССП данного блока, прежде всего, связаны с оптимизацией имеющихся в распоряжении торгового предприятия информационных, производственных, материальных, трудовых, инновационных, интеллектуальных и финансовых ресурсов с учетом внутренних ре- зервов хозяйствующего субъекта для решения текущих задач и стратегических целей.

Традиционно бизнес-процессы включают инновационные процессы (разработка и развитие предлагаемого клиенту продукта, услуги) и операционные (производство, маркетинга, закупочные и сбытовые процессы, логистика, сервис).

Параметры четвертой составляющей - обучения и развития персонала состоят из таких показателей как степень удовлетворенности сотрудников компании, текучесть персонала, уровень квалификации персонала, а также наличие определенных умений и навыков, обеспечивающих компании конкурентное преимущество. Данная составляющая позволяет понять насколько успешно происходит каскадирование стратегических целей компании с управленческого на операционный уровень.

Входящие в ССП показатели являются не просто набором финансовых и нефинансовых индикаторов, разделенных на четыре функциональные группы. Сбалансированность системы предполагает наличие причинно-следственных связей между стратегическими целями и измеряющими их показателями компании, которые изображаются в форме стратегических карт [2].

\section{Библиографический список}

1. Ковалев, В.В.Управление денежными потоками, прибылью и рентабельностью / В.В.Ковалев: учебнопракт. пособие. М: Проспект, 2015.

2. Гибенко Д. С., Минякова Т.Е. Сбалансированная система показателей: сущность, этапы внедрения, основные проблемы // Проблемы современной экономики (Новосибирск). 2010. № 2-2. URL: https://cyberleninka. ru/article/n/sbalansirovannaya-sistema-pokazateley-suschnost-etapy-vnedreniya-osnovnye-problemy (дата обращения: 12.02.2021).

3. Chernysheva N.A., Perskaya V.V., PetrovA.M., Bakulina A.A. GREEN ENERGY FOR BELT AND ROAD INITIATIVE: ECONOMIC ASPECTS TODAY AND IN THE FUTURE / International Journal of Energy Economics and Policy. 2019. T. 9. № 5. C. 178-185.

4. Kevorkova Z.A., Petrov A. M., Savina N. V. TOWARDS LIABILITIES OF CORPORATE SYSTEMS / International Journal of Civil Engineering and Technology. 2019. T. 10. № 2. C. 1582-1593.

5. Petrov A.M., NikiforovaE.V., Kiseleva N.P., Grishkina S.N., LihtarovaO.V. CREATION OF THE REPORTING ON SUSTAINABLE DEVELOPMENT OF COMPANIES BASED ON SOCIOECONOMIC MEASUREMENT STATISTICS / International Journal of Recent Technology and Engineering. 2019. T. 8. № 2. C. 4005-4012.

6. SotnikovaL.V., PolenovaS.N., MislavskayaN.A., PetrovA.M., BasovaM.M. SUSTAINABLE DEVELOPMENT, MACRO AND MICRO LEVEL: RUSSIAN AND FOREIGN MODEL / International Journal of Recent Technology and Engineering. 2019. T. 8. № 2. C. 4524-4532.

7. Kosolapova M.V., Muravitskaya N.K., TolmachevM.N., Melnikova L.A., PetrovA.M. TECHNOLOGY FOR SOLVING THE PROBLEMS RELATED TO THE IMPLEMENTATION OF THE CONCEPT OF PRESERVING CAPITAL IN ACCOUNTING AND STATISTICS / International Journal of Recent Technology and Engineering. 2019. T. 8. № 3. C. 789-792. 
8. Kosolapova M.V., PetrovA.M., YshanovI.G., MuravitskayaN.K., NurmuhamedovaH.S. THE ECONOMIC SIGNIFICANCE OF STATISTICAL RESEARCH ACTIVITIES OF REPRESENTATIVE OFFICES OF COMPANIES ABROAD / International Journal of Innovative Technology and Exploring Engineering. 2019. T. 8. № 10. C. 27132722.

9. Petrov A.M., Yurasova I. O., Putihin Y.E., Poluleh M. V., Erohina V. N. ACCOUNTANT MODELING TECHNOLOGY AND STATISTICS IN THE CONTEXT OF THE NEW EDUCATIONAL CONCEPT / International Journal of Innovative Technology and Exploring Engineering. 2019. T. 8. № 12. C. 3214-3217.

10. Petrov A.M., Kiseleva N.P., KevorkovaZ.A., Melnikova L.A., YshanovI.G. PRESENT DEVELOPMENT PRACTICES FOR TAX, FINANCIAL AND STATISTICAL REPORTING IN THE RUSSIAN FEDERATION / International Journal of Innovative Technology and Exploring Engineering. 2019. T. 8. № 12. C. 3538-3542.

11. Karpova T.P., Petrov A. M., Antonova O. V. DIRECTIONS OF ACCOUNTING DEVELOPMENT IN THE CONDITIONS OF DIGITALIZATION / Journal of Advanced Research in Dynamical and Control Systems. 2018. T. 10. № 7 Special Issue. C. 117-125.

12. Lymar M.P., Kevorkova Z.A., Petrov A. M.THE CONVERGENCE OF NATIONAL AND INTERNATIONAL ACCOUNTING STANDARDS: CHINESE EXPERIENCE / International Journal of Civil Engineering and Technology. 2018. T. 9. № 13. C. $82-94$. 